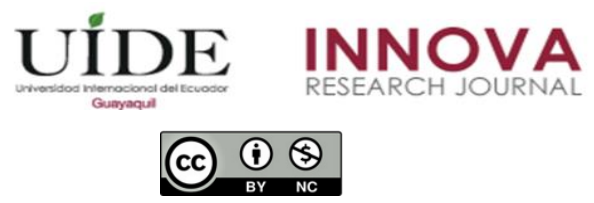

INNOVA Research Journal, ISSN 2477-9024

(Enero-Abril 2021). Vol. 6, No.1 pp.246-256

DOI: https://doi.org/10.33890/innova.v6.n1.2021.1641

URL: http://revistas.uide.edu.ec/index.php/innova/index

Correo: innova@uide.edu.ec

\title{
¿Confinamiento o libre movilidad frente a la pandemia del sars cov 2? Los casos de Suecia, Países Bajos y Brasil
}

\section{Confinement or free movement in sars cov 2 pandemic? The cases of Sweden, The Netherlands and Brazil}

Jorge Andrés Izaguirre Olmedo

https://orcid.org/0000-0001-5178-8641

Universidad Internacional del Ecuador, Ecuador

Autor para correspondencia: joizaguirreol@uide.edu.ec

Fecha de recepción: 1 de noviembre de 2020 - Fecha de aceptación: 21 de diciembre de 2020

\section{Resumen}

Tras la declaratoria de pandemia por parte de la Organización Mundial de la Salud en el 2020 producto del SARS COV 2, los países optaron por medidas que ayuden a controlar el número de contagios y muertes por el virus. Una de las estrategias más utilizadas fue el confinamiento, sin embargo, ciertos países prefirieron no acogerse a dicha estrategia y buscar la inmunidad de grupo. En este artículo se analizan los casos de Suecia, Países Bajos y Brasil y se contrastan sus datos con el promedio de países que si decidieron confinarse. La muestra utilizada cuenta con 16 países, incluyendo los tres casos de estudio. Los resultados señalan que la estrategia de no confinamiento ha presentado cifras de contagios y muertes estadísticamente iguales o menores al promedio que presentan los países que optaron por el confinamiento.

Palabras claves: confinamiento; confinamiento inteligente; inmunidad de grupo; nivel de confianza; SARS COV 2.

\begin{abstract}
After declaring a pandemic by the World Health Organization in 2020 due to the SARS COV 2, countries have opted for measures to help control the number of infections and diseases caused by the virus. One of the most used strategies was confinement, however, certain countries preferred not to agree on this strategy and seek group immunity. This article analyzes the cases of Sweden, the Netherlands and Brazil and contrasts with the data of countries that have decided to confine themselves. The sample includes 16 countries. The results show that the strategy of nonconfinement has reached growth factor and index of diseases statistically equal or lower than countries that have opted for the confinement.
\end{abstract}

Keywords: confinement; smart confinement; group immunity; level of confidence; SARS COV 2. 


\section{Introducción}

El SARS COV 2 es un virus de la familia de coronavirus que tuvo su aparición a finales del 2019 en China (Sanche, y otros, 2020). Debido a la facilidad de contagio, el virus tuvo un rápido crecimiento y se esparció a otros países de Asia y Europa (Tang, y otros, 2020; Bhattacharya \& Singh, 2020) mostrando una tendencia de crecimiento exponencial (Ayoub, y otros, 2020) y características de virus potencialmente mortal.

Frente a la pandemia del SARS COV 2 declarada por la OMS (World Health Organization, 2020), los gobiernos han tomado medidas de prevención y control de la enfermedad con el objetivo de minimizar los efectos en la población (Jia, y otros, 2020). Inicialmente, las medidas correspondían al lavado continuo de manos, evitar contacto con otras personas, estornudar en el propio brazo y evitar contacto de manos con la cara; no obstante, dado el creciente número de casos, los países empezaron a optar por medidas de confinamiento que desaceleren la curva de contagios.

Las medidas de confinamiento tuvieron opiniones a favor y en contra. Las opiniones a favor se basaban en la evolución del virus y su rápido crecimiento ( $\mathrm{Zu}$, y otros, 2020); mientras que las opiniones en contra se basaban en los efectos económicos de la paralización de los negocios (Rosman, y otros, 2020) y en la necesidad de crear una inmunidad de grupo o inmunidad de rebaño (Mayordomo y Moreno, 2020). Frente a esta realidad, ciertos países optaron por medidas alternas al confinamiento, como lo son el confinamiento inteligente, en el cual las personas con mayor riesgo debían auto-confinarse; o el cuidado personal sin medidas de confinamiento. Entre estos países se puede mencionar a Suecia, Países Bajos y Brasil (El Ágora, 2020; Navas, 2020; BBC NEWS MUNDO, 2020).

Transcurridos más de 200 días desde el inicio de la pandemia en los diferentes países afectados, surge la interrogante sobre la efectividad de las políticas o decisiones de los gobiernos frente a la pandemia. En este sentido, este artículo compara los efectos en el crecimiento del número de contagiados y la tasa de mortalidad de Suecia, Países Bajos y Brasil, frente a las cifras de otros países que tomaron medidas de confinamiento obligatorio. El análisis se realiza a través de estimaciones de nivel de confianza al 95\% para la media. Los datos fueron obtenidos de la página worldometers.com en dos instantes de tiempo: a los 60 días luego del primer infectado en cada país y al 14 de octubre del 2020; transcurridos más de 200 días de la pandemia.

\section{Metodología}

Esta investigación corresponde a un estudio descriptivo, de corte seccional, con diseño no experimental. La muestra está constituida por 16 países, de los cuales, 13 corresponden a países que decretaron confinamiento en la pandemia, mientras que 3 países corresponden a aquellos que optaron por confinamiento inteligente o no dictaron medidas de confinamiento alguna.

Los datos fueron obtenidos de Worldometer.com para dos instantes del tiempo. La primera medición corresponde a contagios y muertes en el día 60 después del primer infectado en cada país; mientras que la segunda medición corresponde a las mismas variables para el 14 de

Esta obra se comparte bajo la licencia Creative Common Atribución-No Comercial 4.0 International (CC BY-NC 4.0) Revista de la Universidad Internacional del Ecuador. URL: https://www.uide.edu.ec/ 
octubre del 2020. En este sentido, el estudio se refiere a una medición de corto plazo para la primera medición y mediano plazo para la segunda.

Las variables analizadas corresponden al factor de crecimiento de la pandemia y la tasa de mortalidad. La tasa de mortalidad se calcula por el ratio entre el número de muertos por pandemia entre el número de contagiados; mientras que el factor de crecimiento se computa acorde a la propuesta de Izaguirre y Bazurto (2020) y se muestra en las ecuaciones 1 y 2.

$$
\text { Número de contagios }(f c, t)=(f c)^{t}
$$

Para la obtención del factor de crecimiento se procede al despeje de la variable $f c$. La ecuación 2 muestra la expresión resultante.

$$
f c=\sqrt[t]{\operatorname{casos}}
$$

Dado que el objetivo del estudio es contrastar las cifras de los países con confinamiento frente a aquellos que no presentaron confinamiento obligatorio, inicialmente se planteó un test de medias; no obstante, dado que la muestra cuenta únicamente con tres países para el grupo que corresponde a no confinamiento, se optó por analizar las cifras de cada uno de los países del mencionado grupo frente a las estadísticas del grupo de países con confinamiento, aplicando un nivel de confianza del $95 \%$.

\section{Revisión de literatura}

Ayoub et. al (2000) investigaron las tendencias biológicas y epidemiológicas en la prevalencia y mortalidad debido a brotes de nuevas infecciones por SARS-COV-2. Evaluaron el factor de crecimiento, un factor por el cual la cantidad se multiplica con el tiempo, casos diarios divididos por casos del día anterior. Un factor de crecimiento de más de 1.0 indica un patrón creciente, mientras que los valores entre 0 y 1.0 muestran un patrón de disminución. Como resultado, obtuvieron una tendencia creciente en la transmisión, prevalencia y tasa de mortalidad debido al SARS-COV-2. Dada la creciente transmisión y evolución del virus sin tener una vacuna definida, el camino es la prevención e higiene, el distanciamiento social y las limitaciones de movimiento para controlar la propagación.

Izaguirre y Bazurto (2020) modelaron el factor de crecimiento de la pandemia en su estudio sobre pandemia y corrupción. En su análisis sostienen que el factor de crecimiento de la pandemia varía entre países y está relacionado con el índice de corrupción que tienen los Estados. Señalan también que existe una diferencia en el factor de crecimiento del virus cuando se analizan países de América del Sur.

Tang et. al (2020) investigaron el origen y la evolución continua del SARS-COV-2, utilizando el grado de divergencia molecular entre SARS-COV-2 y otros coronavirus relacionados, obteniendo como resultados la mutación del coronavirus inicial a muchas cepas 
secuenciales. Los autores indican que se deberá continuar analizando nuevas cepas y su evolución en términos de propagación y contagio.

Bhattacharjee (2020) analizó la relación de la propagación del coronavirus y factores ambientales, tomando como variables la humedad relativa máxima, temperatura máxima y la velocidad máxima del viento, evidenciando una insignificante relación entre la humedad relativa máxima y el viento más alto, mientras que con la temperatura máxima muestra que existe una variante entre insignificante y moderada, luego del análisis realizado no existe evidencia significativa que permita concluir que el virus pueda convertirse inactivo en los días de verano.

Li, Romagnani, Brunn y Anders (2000) estudiaron el impacto de la propagación del virus versus la aplicación de cuarentena por país a través del análisis de los casos en China e Italia, considerando la fecha de inicio de cuarentena. Obtuvieron como resultado que el virus creció exponencialmente en cuanto más tarde se implementaron las medidas de aislamiento. El aislamiento se debe implementar al momento de reportar el primer caso de Coronavirus de manera que se pueda controlar la aceleración y crecimiento exponencial del mismo, según concluyen los autores.

Jia et. al (2020) investigaron el impacto de intervenciones políticas y factores ambientales en la aceleración de la pandemia. Utilizaron un modelo de mínimos cuadrados ordinarios y probaron que la cuarentena en el hogar juega un papel importante en el control de la propagación de la enfermedad; resultado que concuerda con las conclusiones que se exponen en esta investigación. Se concluye que la más conveniente estrategia para el control diario de esta enfermedad es la vacunación, sin embargo, el ciclo de desarrollo de la misma es relativamente largo por lo que todavía es necesario el aislamiento como control temprano de la enfermedad.

Mayordomo y Moreno (2020) analizaron las decisiones de los gobiernos frente a la pandemia en lo que respecta a confinamiento o búsqueda de la inmunidad de grupo o rebaño. En su artículo señalan que los países que inicialmente optaron por la inmunidad de rebaño fueron Estados Unidos, Reino Unido, Suecia, Países Bajos y Brasil. No obstante, en Estados Unidos se dejó libertad a los estados para decidir sobre el confinamiento; mientras que, en Reino Unido, la decisión de inmunidad de rebaño cambió rápidamente a confinamiento en los primeros días de pandemia. Por tal motivo, en este estudio se toma a Suecia, Países Bajos y Brasil como casos de estudio de países que optaron por no confinarse.

\section{Resultados}

La muestra contó con 16 países de los cuales 13 pertenecen al grupo de países que tomó medidas de confinamiento ante la pandemia, mientras que 3 de ellos optaron por medidas de confinamiento inteligente o, simplemente, ninguna medida al respecto. La tabla 1 presenta los detalles de tasa de mortalidad y factor de crecimiento de contagios para los países de la muestra. 


\section{Tabla 1}

Tasa de mortalidad y factor de crecimiento de la pandemia por países de la muestra

\begin{tabular}{lcccc}
\hline País & $\begin{array}{c}\text { Tasa mortalidad 14 } \\
\text { de octubre }\end{array}$ & $\begin{array}{c}\text { Factor de } \\
\text { crecimiento } \\
\text { octubre }\end{array}$ & $\begin{array}{c}\text { Tasa } \\
\text { mortalidad 60 } \\
\text { días }\end{array}$ & $\begin{array}{c}\text { Factor de } \\
\text { crecimiento 60 } \\
\text { días }\end{array}$ \\
\hline España & $3,48 \%$ & $\begin{array}{c}\text { Países con confinamiento } \\
\text { Reino }\end{array}$ & 1,055 & 1,222 \\
Unido & $6,59 \%$ & 1,056 & $16,22 \%$ & 1,207 \\
Italia & $9,73 \%$ & 1,051 & $11,43 \%$ & 1,212 \\
Francia & $4,24 \%$ & 1,053 & $4,93 \%$ & 1,182 \\
Alemania & $2,86 \%$ & 1,050 & $0,69 \%$ & 1,198 \\
Perú & $3,91 \%$ & 1,063 & $7,19 \%$ & 1,198 \\
Chile & $2,76 \%$ & 1,060 & $1,29 \%$ & 1,181 \\
México & $10,23 \%$ & 1,061 & $9,23 \%$ & 1,175 \\
Ecuador & $8,23 \%$ & 1,054 & $7,16 \%$ & 1,179 \\
Suiza & $3,07 \%$ & 1,049 & $5,53 \%$ & 1,187 \\
Colombia & $3,04 \%$ & 1,064 & $4,39 \%$ & 1,163 \\
Argentina & $2,67 \%$ & 1,063 & $13,49 \%$ & 1,152 \\
Uruguay & $2,14 \%$ & 1,037 & $2,65 \%$ & 1,116 \\
Brasil & Países con confinamiento inteligente o sin confinamiento & \\
Suecia & $2,95 \%$ & 1,069 & $6,83 \%$ & 1,201 \\
Países & $5,77 \%$ & 1,049 & $13,24 \%$ & 1,170 \\
Bajos & $3,40 \%$ & 1,054 & $11,81 \%$ & 1,192 \\
\hline
\end{tabular}

Fuente: Worldometers.com

Elaboración: El autor

Los datos fueron obtenidos en dos instantes del tiempo. Una primera observación corresponde a los 60 días después de identificado el primer contagiado en cada país. La segunda observación corresponde al 14 de octubre del 2020, más de 200 días después de la pandemia en todos los países. Al comparar las cifras se podrán observar cifras en el corto plazo, identificado por los 6 días; y el mediano plazo, identificado por la segunda observación.

Como puede observarse, para todos los casos de la muestra se presenta una disminución en el factor de crecimiento de contagios desde la primera hasta la segunda observación. Podría suponerse que esta disminución tenga múltiples factores: medidas de confinamiento, aprendizaje de la población sobre los cuidados en pandemia, tamaño de la población contagiada, entre otros. 


\section{Tabla 2}

Estadísticas descriptivas para países con confinamiento

\begin{tabular}{lcccc}
\hline & $\begin{array}{c}\text { Tasa } \\
\text { mortalidad 14 } \\
\text { de octubre }\end{array}$ & $\begin{array}{c}\text { Factor de } \\
\text { crecimiento 14 } \\
\text { de octubre }\end{array}$ & $\begin{array}{c}\text { Tasa } \\
\text { mortalidad } \\
\mathbf{6 0 ~ d i ́ a s}\end{array}$ & $\begin{array}{c}\text { Factor de } \\
\text { crecimiento 60 } \\
\text { días }\end{array}$ \\
\hline Media & $4,84 \%$ & 1,055 & $6,86 \%$ & 1,182 \\
Mediana & $3,48 \%$ & 1,055 & $5,53 \%$ & 1,182 \\
Mínimo & $2,14 \%$ & 1,037 & $0,69 \%$ & 1,116 \\
Máximo & $10,23 \%$ & 1,064 & $16,22 \%$ & 1,222 \\
Nivel de confianza & $1,72 \%$ & 0,005 & $2,81 \%$ & 0,017 \\
$(95,0 \%)$ & & & & \\
\hline
\end{tabular}

Fuente: Worldometers.com

Elaboración: El autor

La tabla 2 presenta las estadísticas descriptivas para los países que optaron por medidas de confinamiento. Tanto la media como la mediana muestran que el factor de crecimiento de la pandemia pasó de 1.182 a 1.055 desde el corto plazo hasta el mediano plazo. Así mismo, el nivel máximo que se ubicó en 1.222 a inicios de la pandemia, luego disminuyó a 1.064, mostrando una clara reducción de la velocidad de crecimiento de la pandemia. Se observa también que la tasa de mortalidad disminuyó alrededor de dos puntos porcentuales, lo cual podría explicarse por nuevos métodos para tratar a los infectados y reducir el riesgo de muerte. Inicialmente, el nivel máximo de mortalidad se ubicó $16.22 \%$, sin embargo, en el mediano plazo el máximo se presenta en $10.23 \%$.

\section{Suecia}

Suecia fue uno de los países que optó por no confinar a su población durante la pandemia (Navas, 2020). La intención de esta medida es apelar a lo que se conoce como la inmunidad de grupo o inmunidad de rebaño, considerando que el virus iba a estar presente de cualquier manera. La tabla 3 muestra una comparativa de las cifras de Suecia frente al promedio de los países que realizaron confinamiento. Los valores mínimos y máximos corresponden al 95\% de confianza de la media. 
Tabla 3

Resultados de Suecia frente a países con confinamiento

\begin{tabular}{lcccc}
\hline & $\begin{array}{c}\text { Tasa mortalidad } \\
\mathbf{1 4} \text { de octubre }\end{array}$ & $\begin{array}{c}\text { Factor de crecimiento } \\
\mathbf{1 4} \text { de octubre }\end{array}$ & $\begin{array}{c}\text { Tasa mortalidad } \\
\mathbf{6 0} \text { días }\end{array}$ & $\begin{array}{c}\text { Factor de } \\
\text { crecimiento 60 días }\end{array}$ \\
\hline $\begin{array}{l}\text { Valor mínimo } \\
\text { de la media }\end{array}$ & $3,12 \%$ & 1,051 & $4,04 \%$ & 1,165 \\
$\begin{array}{l}\text { Valor máximo } \\
\text { de la media }\end{array}$ & $6,56 \%$ & 1,060 & $9,67 \%$ & 1,199 \\
Suecia & $5,77 \%$ & $\mathbf{1 , 0 4 9}$ & $\mathbf{1 3 , 2 4 \%}$ & 1,170 \\
\hline
\end{tabular}

Fuente: Worldometers

Elaboración: El autor

Según se observa en la tabla, en los 60 días de iniciada la pandemia, Suecia presenta un factor de crecimiento de contagios que no es estadísticamente distinto al factor de crecimiento de los países con confinamiento; mientras que, al 14 de octubre se observa que el factor de crecimiento de contagios se ubica en 1.049, estadísticamente menor que el promedio de los países con confinamiento. Esta estadística sugiere que la estrategia de no confinamiento de Suecia fue efectiva en términos de crecimiento de contagios.

Por otro lado, se observa también que la tasa de mortalidad inicial para Suecia fue de $13.24 \%$, estadísticamente superior a la tasa de mortalidad observada en países con confinamiento; sin embargo, en el mediano plazo, dicha tasa de mortalidad disminuyó y se ubicó en el rango promedio de los países que confinaron a su población. En otras palabras, aunque inicialmente la tasa de mortalidad de Suecia fue más alta, con el paso del tiempo no es distinta a los otros países.

\section{Países Bajos}

Países Bajos fue otro de los países que optó por confinamiento inteligente (Semana, 2020). Esta estrategia otorga libertad a la población para confinarse acorde a su nivel de riesgo frente al virus. La estrategia ha sido considerada como de alto riesgo, no obstante, la tabla 4 presenta las cifras observadas en el corto y mediano plazo.

\section{Tabla 4}

Resultados de Países Bajos frente a países con confinamiento

\begin{tabular}{lcccc}
\hline & $\begin{array}{c}\text { Tasa mortalidad } \\
\mathbf{1 4} \text { de octubre }\end{array}$ & $\begin{array}{c}\text { Factor de crecimiento } \\
\mathbf{1 4} \text { de octubre }\end{array}$ & $\begin{array}{c}\text { Tasa mortalidad } \\
\mathbf{6 0} \text { días }\end{array}$ & $\begin{array}{c}\text { Factor de } \\
\text { crecimiento 60 días }\end{array}$ \\
\hline $\begin{array}{l}\text { Valor mínimo } \\
\text { de la media }\end{array}$ & $3,12 \%$ & 1,051 & $4,04 \%$ & 1,165 \\
Valor máximo & $6,56 \%$ & 1,060 & $9,67 \%$ & 1,199 \\
de la media & $3,40 \%$ & 1,054 & $\mathbf{1 1 , 8 1 \%}$ & 1,192 \\
\hline Países Bajos & & &
\end{tabular}

Fuente: Worldometers.com

Elaboración: El autor 
Acorde a los datos, al inicio de la pandemia el factor de crecimiento de Países Bajos no es estadísticamente distinto al factor que presentan países con confinamiento; situación que se observó también para el caso de Suecia. En el mediano plazo, el factor de crecimiento de Países bajos es de 1.054, que sigue manteniéndose en el promedio de países confinados; por tanto, no existe evidencia estadística para asegurar que la estrategia de confinamiento inteligente de Países Bajos ha generado mayor cantidad de contagios que países con confinamiento.

En lo que respecta a la mortalidad, se observa que, al igual que sucedía con Suecia, Países Bajos presenta una tasa de mortalidad estadísticamente mayor que otros países a inicios de la pandemia. No obstante, si se analizan las cifras en el mediano plazo, la tasa de mortalidad de Países Bajos se ubica en el rango de confianza de la media de los países confinados; por lo que puede concluirse que, en el mediano plazo, la estrategia de confinamiento inteligente del país de análisis alcanzó el mismo resultado de aquellos países con medidas restrictivas.

\section{Brasil}

En lo que respecta a Sudamérica, Brasil no tuvo clara una postura de confinamiento. Las declaraciones del presidente de dicho país estuvieron inclinadas por un mantenimiento de la economía a pesar de la pandemia que estaba iniciando. Hasta octubre del 2020, Brasil es uno de los países que presenta mayor nivel de contagios y muertes por coronavirus a nivel mundial (BBC NEWS MUNDO, 2020). La tabla 5 presenta el comparativo de Brasil con respecto a los otros países.

\section{Tabla 5}

Resultados de Brasil frente a países con confinamiento

\begin{tabular}{lcccc}
\hline & $\begin{array}{c}\text { Tasa mortalidad } \\
\text { 14 de octubre }\end{array}$ & $\begin{array}{c}\text { Factor de } \\
\text { crecimiento } \\
\mathbf{1 4} \text { de octubre }\end{array}$ & $\begin{array}{c}\text { Tasa } \\
\text { mortalidad } \\
\mathbf{6 0} \text { días }\end{array}$ & $\begin{array}{c}\text { Factor de } \\
\text { crecimiento 60 días }\end{array}$ \\
\hline $\begin{array}{l}\text { Valor mínimo } \\
\text { de la media }\end{array}$ & $3,12 \%$ & 1,051 & $4,04 \%$ & 1,165 \\
$\begin{array}{l}\text { Valor máximo } \\
\text { de la media }\end{array}$ & $6,56 \%$ & 1,060 & $9,67 \%$ & 1,199 \\
Brasil & $\mathbf{2 , 9 5 \%}$ & $\mathbf{1 , 0 6 9}$ & $6,83 \%$ & $\mathbf{1 , 2 0 1}$ \\
\hline
\end{tabular}

Fuente: Worldometers.com

Elaboración: El autor

La tabla 5 muestra que el factor de crecimiento de la pandemia en Brasil, tanto en el corto como en el mediano plazo, es estadísticamente mayor que el factor de crecimiento de países con medidas de confinamiento. En este sentido, la estrategia de libre movilidad optada por Brasil ha presentado resultados desfavorables en términos de crecimiento del número de contagiados.

Por otra parte, se observa también la tasa de mortalidad en el corto y mediano plazo. A pesar de que en el corto plazo el factor de crecimiento de contagios de Brasil es mayor, la tasa de 
mortalidad que presenta no es estadísticamente distinta a la tasa de mortalidad que se presenta en países confinados. Sin embargo, si se observa la tasa de mortalidad de mediano plazo, ésta asciende a $2.95 \%$, lo cual es estadísticamente menor que la tasa de mortalidad de la muestra. Por tanto, a pesar de tener un crecimiento mayor en contagiados, presenta una tasa de muerte menor. Este estudio no aborda las razones por las que la tasa de mortalidad en Brasil disminuyó, sin embargo, podría plantearse como hipótesis la obtención de inmunidad de grupo que se analiza en el marco teórico.

\section{Conclusión}

En base a la información presentada, se procede a exponer las conclusiones del estudio. El objetivo inicial fue contrastar las cifras de crecimiento de la pandemia y tasa de mortalidad para países que optaron por medidas de confinamiento inteligente o libertad de movilidad, frente a las cifras de países que optaron por el confinamiento obligatorio. Para el efecto, se trabajó con una muestra de 13 países que optaron por el confinamiento y se obtuvieron estadísticas descriptivas para contrastarse con las cifras de Suecia, Países Bajos y Brasil.

Acorde a los datos, se observa que tanto el factor de crecimiento de contagios como la tasa de mortalidad se han reducido desde los 60 días desde el primer contagiado en cada país, hasta la segunda medición con fecha 14 de octubre. Este estudio no aborda las razones para dicha disminución, pero entre las hipótesis se puede mencionar un aprendizaje para manejar los casos de contagio y el cuidado de la población como método preventivo.

En lo que respecta a Suecia y Países Bajos, ambos presentaron tasas de mortalidad iniciales que eran estadísticamente mayores que las tasas de mortalidad de países confinados; no obstante, el factor de crecimiento presentado no fue estadísticamente distinto. Sin embargo, al analizar las cifras de mediano plazo, la tasa de mortalidad se ubicó en niveles que mantienen los países que optaron por confinamiento; lo cual implica que la estrategia utilizada por Suecia y Países Bajos, tiene resultados estadísticamente iguales que los otros países. Incluso, el factor de crecimiento de la pandemia para Suecia es menor en el mediano plazo que el promedio de los países con confinamiento; sugiriendo así una posible efectividad de inmunidad de grupo o rebaño.

Por otra parte, el factor de crecimiento de la pandemia en Brasil, tanto en el corto como en el mediano plazo, es estadísticamente mayor que el factor de crecimiento de países con medidas de confinamiento. Sin embargo, la tasa de mortalidad en el corto plazo no es estadísticamente distinta a los países con restricciones; mientras que la tasa de mortalidad en el mediano plazo se muestra estadísticamente menor. Este resultado muestra que la estrategia utilizada por Brasil ha sido efectiva en términos de mortalidad, mas no en términos de número de contagiados. En este sentido, el caso de Brasil sustenta la teoría de la inmunidad de grupo o rebaño. 


\section{Referencias Bibliográficas}

Ayoub, S., Al-Khlaiwia, T., Mahmood, A., Sultan, A., Klonoff, D., \& Hoang, T. (2020). Biological and epidemiological trends in the prevalence and mortality due to outbreaks of novel coronavirus COVID-19. Journal of King Saud University - Science, https://www.sciencedirect.com/science/article/pii/S1018364720301518.

BBC NEWS MUNDO. (18 de 05 de 2020). Coronavirus en Brasil: 7 errores que llevaron a Brasil a la crítica situación actual. BBC NEWS, págs. https://www.bbc.com/mundo/noticias-america-latina-52708003.

Bhattacharjee, S. (2020). Statistical investigation of relationship between spread of coronavirus disease (COVID19) and environmental factors based on study of four mostly affected places of China and five mostly affected places of Italy. The Assam Royal Global University, https://arxiv.org/ftp/arxiv/papers/2003/2003.11277.pdf.

Bhattacharya, S., \& Singh, S. (2020). Visible Insights of the Invisible Pandemic: A Scientometric, Altmetric and Topic Trend Analysis. CSIR-National Institute of Science Technology and Development Studies, https://arxiv.org/abs/2004.10878.

El Ágora. (06 de 04 de 2020). De Bielorrusia a Suecia: los países menos restrictivos con el coronavirus. Obtenido de https://www.elagoradiario.com/coronavirus/diariointernacional/bielorrusia-suecia-paises-menos-restrictivos-coronavirus/

Izaguirre, J., \& Bazurto, E. (2020). La corrupción y su impacto en el crecimiento de la pandemia sars-cov-2 en sudamérica. RES NON VERBA, 39 - 51.

Jia, J., Ding, J., Liu, S., Liao, G., Li, J., Duan, B., . . Z Zhang, R. (2020). Modeling the Control of COVID-19: Impact of Policy Interventions and Meteorological Factors. Cornell University, https://arxiv.org/abs/2003.02985.

Lewnarda, J., \& Lo, N. (2020). Scientific and ethical basis for social-distancing interventions against COVID-19. Elsevier Public Health Emergency Collection, https://www.ncbi.nlm.nih.gov/pmc/articles/PMC7118670/.

Li, C., Romagnani, P., Brunn, A.-V., \& Anders, H.-J. (2020). SARS-CoV-2 and Europe: timing of containment measures for outbreak control. US National Library of Medicine National Institutes of Health, https://www.ncbi.nlm.nih.gov/pmc/articles/PMC7142270/.

Liu, Z., Zhang, Z., Wang, X., Gong, H., Liu, X., Chen, H., . . Gao, C. (2020). Daily Tracking and Forecasting of the Global COVID-19 Pandemic Trend Using Holt-Winters Exponential Smoothing. Obtenido de https://papers.ssrn.com/sol3/papers.cfm?abstract_id=3564413\&download=yes

Mayordomo, C., \& Moreno, S. (2020). De la inmunidad al confinamiento. Dos paradigmas de una misma crisis. Más poder local. N 41, 42 - 43.

Navas, M.-E. (18 de 05 de 2020). Coronavirus en Suecia: el debate que despierta la singular estrategia del país europeo de no confinar a su población durante la pandemia. $B B C$ NEWS, págs. https://www.bbc.com/mundo/noticias-internacional-52690735.

Rosman, T., Chasiotis, A., Kerwer, M., Steinmetz, H., Wedderhoff, O., \& Bosnjak, M. (2020). Will COVID-19-related economic worries superimpose the health worries, reducing acceptance of social distancing measures? A prospective pre-registered study. ZPID (Leibniz Institute for Psychology Information), http://dx.doi.org/10.23668/psycharchives.2803. 
Sanche, S., Lin, Y. T., Xu, C., Romero-Severson, E., Hengartner, N., \& Ke, R. (2020). High Contagiousness and Rapid Spread of Severe Acute Respiratory Syndrome Coronavirus 2. Emerg Infect Dis. Volume 26, Number 7, https://doi.org/10.3201/eid2607.200282.

Semana. (15 de 10 de 2020). Coronavirus: por qué el "confinamiento inteligente" de Países Bajos puede ser una estrategia de alto riesgo. Obtenido de https://www.semana.com/mundo/articulo/coronavirus-por-que-el-confinamientointeligente-de-paises-bajos-puede-ser-una-estrategia-de-alto-riesgo/661975/

Tang, X., Wu, C., Li, X., Song, Y., Yao, X., Wu, X., . . Lu, J. (2020). On the origin and continuing evolution of SARS-CoV-2. National Science Review, https://academic.oup.com/nsr/advance-article/doi/10.1093/nsr/nwaa036/5775463.

World Health Organization. (27 de 05 de 2020). Coronavirus disease (COVID-19) Pandemic. Obtenido de https://www.who.int/emergencies/diseases/novel-coronavirus-2019

Zu, J., Li, M., Li, Z., Shen, M., Xiao, Y., \& Ji, F. (2020). Epidemic trend and transmission risk of SARS-CoV-2 after government intervention in the mainland of China: A mathematical model study. Obtenido de https://papers.ssrn.com/sol3/papers.cfm?abstract_id=3539669 